Open J. Math. Anal., Vol. 1(2017), No. 1, pp. $34-43$

Website: https://pisrt.org/psr-press/journals/oma/

ISSN: 2616-8111(Online), 2616-8103 (Print)

http://dx.doi.org/10.30538/psrp-oma2017.0004

\title{
MAPPING PROPERTIES OF INTEGRAL OPERATOR INVOLVING SOME SPECIAL FUNCTIONS
}

\author{
MUHEY U DIN ${ }^{1}$, MOHSAN RAZA, SADDAF NOREEN
}

\begin{abstract}
In this article, we are mainly interested to find some sufficient conditions for integral operator involving normalized Struve and Dini function to be in the class $N(\mu)$. Some corollaries involving special functions are also the part of our investigations.
\end{abstract}

AMS Mathematics Subject Classification: 30C45, 33C10, 30C20, 30C75.

Key words and phrases: analytic functions; open unit disc; Bessel function; Struve function; Dini function; Salagean derivative.

\section{Introduction}

Let $\mathcal{A}$ denote the class of functions $f$ of the form

$$
f(z)=z+\sum_{n=2}^{\infty} a_{n} z^{n}
$$

which are analytic in the open unit $\operatorname{disc} \mathcal{U}=\{z:|z|<1\}$ and $\mathcal{S}$ denote the class of all functions in which are univalent in $\mathcal{U}$. Let $\mathcal{S}^{*}(\alpha), \mathcal{C}(\alpha)$ and $\mathcal{K}(\alpha)$ denote the classes of starlike, convex and close-to-convex functions of order $\alpha$ and are defined as:

$$
\begin{gathered}
\mathcal{S}^{*}(\alpha)=\left\{f: f \in \mathcal{A} \text { and } \operatorname{Re}\left(\frac{z f^{\prime}(z)}{f(z)}\right)>\alpha, \quad z \in \mathcal{U}, \alpha \in[0,1)\right\} \\
\mathcal{C}(\alpha)=\left\{f: f \in \mathcal{A} \text { and } \operatorname{Re}\left(1+\frac{z f^{\prime \prime}(z)}{f^{\prime}(z)}\right)>\alpha, \quad z \in \mathcal{U}, \alpha \in[0,1)\right\},
\end{gathered}
$$

Received 08-21-2017. Revised 11-25-2017.

1 Corresponding Author

(C) 2017 Muhey U Din, Mohsan Raza, Saddaf Noreen. This is an open access article distributed under the Creative Commons Attribution License, which permits unrestricted use, distribution, and reproduction in any medium, provided the original work is properly cited. 
and

$$
\mathcal{K}(\alpha)=\left\{f: f \in \mathcal{A} \text { and } \operatorname{Re}\left(\frac{z f^{\prime}(z)}{g(z)}\right)>\alpha, \quad z \in \mathcal{U}, \alpha \in[0,1), g \in \mathcal{S}^{*}\right\} .
$$

It is clear that

$$
\mathcal{S}^{*}(0)=\mathcal{S}^{*}, \mathcal{C}(0)=\mathcal{C} \text { and } \mathcal{K}(0)=\mathcal{K} .
$$

Here, we introduce another class $N(p, \gamma), 1<\gamma<\frac{2^{p}+1}{2^{p-1}+1}$, consisting of the function of the form of (1) satisfying the relation

$$
\operatorname{Re}\left(\frac{D^{p+1} f_{j}(z)}{D^{p} f_{j}(z)}\right)<\gamma,
$$

where $D^{p}$ stands for the Salagean operator introduced by Salagean [1] in 1983 . For $p=0, p=1$ the class $N(p, \gamma)$ reduce to the classes $M(\gamma)$ and $N(\gamma)$, respectively. These classes were generalized by many researchers. For the study of these classes we refer [2].

In geometric function theory, special functions play an important role. Special functions have their own importance in pure and applied mathematics. The widely use of these functions have attracted many researchers to work on the different directions. Geometric properties of special functions such as Hypergeometric functions, Bessel functions, Struve functions, Mittage Lefler functions, Wright functions and some other related functions is an ongoing part of research in geometric function theory. We refer for some geometric properties of these functions [3, 4, 5, 6, 7, 8, 9, 10, and references therein.

The Struve functions $H_{v}$ and $L_{v}$ appeared as special solutions of the second order inhomogeneous differential equations of the form

$$
\begin{aligned}
& z^{2} w^{\prime \prime}(z)+z w(z)+\left(z^{2}-v^{2}\right) w(z)=\frac{4\left(\frac{1}{2} z\right)^{v+1}}{\sqrt{\pi} \Gamma\left(v+\frac{1}{2}\right)}, \\
& z^{2} w^{\prime \prime}(z)+z w(z)-\left(z^{2}-v^{2}\right) w(z)=\frac{4\left(\frac{1}{2} z\right)^{v+1}}{\sqrt{\pi} \Gamma\left(v+\frac{1}{2}\right)},
\end{aligned}
$$

known as inhomogeneous Bessel differential equations. Both equations (2) and (3) are similar and can be converted into each other by changing $z$ into $i z$. In the solution of equation (2), a function appeared in an article by Struve [11, was later ascribed Struve's name and the special notation $H_{v}$. It is defined as

$$
H_{v}(z)=\sum_{n=0}^{\infty} \frac{(-1)^{n}\left(\frac{z}{2}\right)^{2 n+v+1}}{\Gamma\left(n+\frac{3}{2}\right) \Gamma\left(n+v+\frac{3}{2}\right)} .
$$

The modified Struve functions $L_{v}$ of order $v$ was introduced by J. W. Nicholson in 1911. It is defined as

$$
L_{v}(z)=-i e^{-i v \frac{\pi}{2}} H_{v}(i z)=\sum_{n=0}^{\infty} \frac{\left(\frac{z}{2}\right)^{2 n+v+1}}{\Gamma\left(n+\frac{3}{2}\right) \Gamma\left(n+v+\frac{3}{2}\right)},
$$


where $\Gamma(z)$ is the gamma function. Applications of Struve functions occur in water-wave and surface-wave problems, unsteady aerodynamics, resistive MHD instability theory and optical diffraction. More recently, Struve functions have appeared in many particle quantum dynamical studies of spin decoherence and nanotubes.

Now consider the second order inhomogeneous differential equation

$$
z^{2} w^{\prime \prime}(z)+b z w^{\prime}(z)+\left[c z^{2}-v^{2}+(1-b) v\right] w(z)=\frac{4\left(\frac{z}{2}\right)^{v+1}}{\sqrt{\pi} \Gamma\left(v+\frac{b}{2}\right)},
$$

where $b, c, v \in \mathbb{C}$. The equation (6) generalizes the equation (2) and (3). In particular for $b=1, c=1$, we obtain (2). For $b=1, c=-1$ we get (3). Its particular solution has the series form

$$
w_{v}(z)=\sum_{n=0}^{\infty} \frac{(-1)^{n} c^{n}\left(\frac{1}{2} z\right)^{2 n+v+1}}{\Gamma\left(n+\frac{3}{2}\right) \Gamma\left(n+v+\frac{b+2}{2}\right)} .
$$

It is known as generalized Struve functions of order $v$. Consider the transformation

$$
\begin{aligned}
u_{v, b, c}(z)= & 2^{v} \sqrt{\pi} \Gamma(v+(b+2) / 2) z^{(-v-1) / 2} w_{v, b, c}(\sqrt{z}) \\
= & \sum_{k=0}^{\infty} \frac{(-c / 4)^{n} z^{n}}{(3 / 2)_{n}(k)_{n}}
\end{aligned}
$$

where $k=v+(b+2) / 2 \neq 0,-1,-2,-3, \ldots$ and

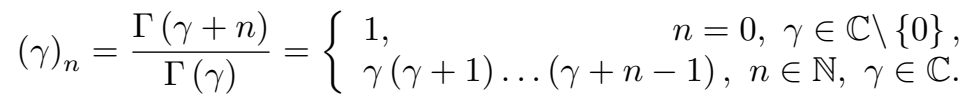

The function $u_{v, b, c}$ is analytic in $\mathcal{U}$ and is the solution of the differential equation

$$
4 z^{2} u^{\prime \prime}(z)+2(2 v+b+3) z u^{\prime}(z)+(c z+2 v+b) u(z)=2 v+b .
$$

The Bessel function of the first kind $J_{v}$ is defined by

$$
J_{v}(z)=\sum_{m=1}^{\infty} \frac{(-1)^{m}}{m ! \Gamma(v+m+1)}\left(\frac{z}{2}\right)^{2 m+v},
$$

where $\Gamma$ stands for Euler gamma function. It is a particular solution of the second order linear homogeneous differential equation

$$
z^{2} w^{\prime \prime}(z)+z w^{\prime}(z)+\left(z^{2}-v^{2}\right) w(z)=0
$$

where $v \in \mathbb{C}$. We consider the normalized Dini functions $q_{v}: \mathcal{U} \rightarrow \mathbb{C}$ defined as

$$
\begin{aligned}
q_{v}(z) & =2^{v-1} \Gamma(v+1) z^{1-\frac{v}{2}}\left((2-v) J_{v}(\sqrt{z})+\sqrt{z} J_{v}^{\prime}(\sqrt{z})\right) \\
& =z+\sum_{m=1}^{\infty} \frac{(-1)^{m}(m+1) \Gamma(v+1)}{4^{m} m ! \Gamma(v+m+1)} z^{m+1}, z \in \mathcal{U}
\end{aligned}
$$


For normalized Dini functions we refer [12, 13. In the present paper, we are mainly interested about the integral operator involving some special functions defined as

$$
F_{v_{1}, \ldots, v_{n}, \alpha_{1}, \ldots, \alpha_{n}, \beta_{1}, \ldots, \beta_{m}}(z)=\lim _{0}^{z} \prod_{i=1}^{n}\left(\frac{g(t)}{t}\right)^{\alpha_{i}} \prod_{i=1}^{n}\left(\frac{D^{p} f_{j}(t)}{t}\right)^{\beta_{j}} d t .
$$

This integral operator generalizes many operators due to this reason it has great importance. By assigning different values to parameters we get differenr operators defined in [14, 15, 16, 17. Recently Porwal and kumar [18, studied the mapping properties of integral operators involvig Bessel functions.

\section{Main Lemmas}

The following lemmas play an very important role to derive our main results.

Lemma 2.1. 6] If $b, v \in \mathbb{R}$, and $c \in \mathbb{C}, k=v+\frac{b+2}{2}$ are so constrained that

$$
k>\max \left\{0, \frac{7|c|}{24}\right\},
$$

then the function $u_{v, b, c}: \mathcal{U} \rightarrow \mathbb{C}$ defined by (8) satisfies the following inequality:

$$
\left|\frac{z u_{v, b, c}^{\prime}(z)}{u_{v, b, c}(z)}-1\right| \leq \frac{|c|(6 k-|c|)}{3(4 k-|c|)(3 k-|c|)} \quad(z \in \mathcal{U}) .
$$

Lemma 2.2. 12] Let $v \in \mathbb{R}$ and consider the normalized Dini function $q_{v}(z)$ : $\mathcal{U} \rightarrow \mathbb{C}$, defined by

$$
q_{v}(z)=2^{v-1} \Gamma(v+1) z^{1-\frac{v}{2}}\left((2-v) J_{v}(\sqrt{z})+\sqrt{z} J_{v}^{\prime}(\sqrt{z})\right),
$$

where $J_{v}(z)$ is the Bessel function of first kind. Then

$$
\left|\frac{z q_{v}^{\prime}(z)}{q_{v}(z)}-1\right| \leq \frac{4 v+9}{2\left(4 v^{2}+9 v+3\right)}, \quad v>\frac{-9+\sqrt{33}}{8} .
$$

The main objective of this paper is to give sufficient conditions for integral operator involving some special functions. The main results are given below.

\section{Sufficient Conditions of Integral Operator Defined by Normalized Struve Function}

Theorem 3.1. Let $v_{1}, \ldots, v_{n}, b \in \mathbb{R}, c \in \mathbb{C}$ and $k_{i}>\frac{7|c|}{24}$ with $k_{i}=v_{i}+$ $(b+2) / 2, i=1, \ldots, n$. Let $u_{v_{i}, b, c}: \mathcal{U} \rightarrow \mathbb{C}$ be defined as

$$
u_{v_{i}, b, c}(z)=2^{v} \sqrt{\pi} \Gamma\left(v+\frac{b+2}{2}\right) z^{\frac{(-1-v)}{2}} w_{v_{i}, b, c}(\sqrt{z}) .
$$

Suppose $k=\min \left\{k_{1}, k_{2}, \ldots, k_{n}\right\}, \alpha_{1}, \ldots, \alpha_{n}, \beta_{1}, \ldots, \beta_{m}(i=1,2, \ldots, n)$ are positive real numbers and let $f_{j}(z),(j=1,2, \ldots, m)$ be of the form of (1) is in class 
$N\left(p, \gamma_{j}\right)$. More over these numbers satisfy the relation

$$
1<1+\frac{|c|(6 k-|c|)}{3(4 k-|c|)(3 k-|c|)} \sum_{i=1}^{n} \alpha_{i}+\sum_{j=1}^{m} \beta_{j}\left(\gamma_{j}-1\right)<\frac{2^{p}+1}{2^{p-1}+1},
$$

then the function $F_{v_{1}, \ldots, v_{n}, \alpha_{1}, \ldots, \alpha_{n}, \beta_{1}, \ldots, \beta_{m}}: \mathcal{U} \rightarrow \mathbb{C}$ defined by 12 is in $N(\mu)$, where

$$
\mu=1+\frac{|c|(6 k-|c|)}{3(4 k-|c|)(3 k-|c|)} \sum_{i=1}^{n} \alpha_{i}+\sum_{j=1}^{m} \beta_{j}\left(\gamma_{j}-1\right) .
$$

Proof. We easily observe that $u_{v_{i}, b, c}, \forall i=1,2, \cdots n$ are analytic and normalized of the form of $u_{v_{i}, b, c}(0)=u_{v_{i}, b, c}^{\prime}(0)-1=0$. Clearly $F_{v_{1}, \ldots, v_{n}, \alpha_{1}, \ldots, \alpha_{n}, \beta_{1}, \ldots, \beta_{m}}$ also analytic and normalized form of the form of $F_{v_{1}, \ldots, v_{n}, \alpha_{1}, \ldots, \alpha_{n}, \beta_{1}, \ldots, \beta_{m}}(0)=$ $F_{v_{1}, \ldots, v_{n}, \alpha_{1}, \ldots, \alpha_{n}, \beta_{1}, \ldots, \beta_{m}}^{\prime}(0)-1=0$. On the other hand, it is easy to see that

$$
F_{v_{1}, \ldots, v_{n}, \alpha_{1}, \ldots, \alpha_{n}, \beta_{1}, \ldots, \beta_{m}}(z)=\prod_{i=1}^{n}\left(\frac{u_{v_{i}, b, c}}{z}\right)^{\alpha_{i}} \prod_{i=1}^{n}\left(\frac{D^{p} f_{j}(z)}{z}\right)^{\beta_{j}} .
$$

Differentiating logarithmically, we get

$$
\begin{aligned}
& \frac{z F_{v_{1}, \ldots, v_{n}, \alpha_{1}, \ldots, \alpha_{n}, \beta_{1}, \ldots, \beta_{m}}^{\prime \prime}(z)}{F_{v_{1}, \ldots, v_{n}, \alpha_{1}, \ldots, \alpha_{n}, \beta_{1}, \ldots, \beta_{m}}^{\prime}(z)} \\
& =\sum_{i=1}^{n} \alpha_{i}\left(\frac{z u_{v_{i}, b, c}^{\prime}(z)}{u_{v_{i}, b, c}(z)}-1\right)+\sum_{j=1}^{m} \beta_{j}\left(\frac{D^{p+1} f_{j}(z)}{D^{p} f_{j}(z)}-1\right),
\end{aligned}
$$

or equivalently,

$$
\begin{aligned}
& 1+\frac{z F_{v_{1}, \ldots, v_{n}, \alpha_{1}, \ldots, \alpha_{n}, \beta_{1}, \ldots, \beta_{m}}^{\prime \prime}(z)}{F_{v_{1}, \ldots, v_{n}, \alpha_{1}, \ldots, \alpha_{n}, \beta_{1}, \ldots, \beta_{m}}^{\prime}(z)} \\
& =\sum_{i=1}^{n} \alpha_{i}\left(\frac{z u_{v_{i}, b, c}^{\prime}(z)}{u_{v_{i}, b, c}(z)}\right)+\sum_{j=1}^{m} \beta_{j}\left(\frac{D^{p+1} f_{j}(z)}{D^{p} f_{j}(z)}\right)+1-\sum_{i=1}^{n} \alpha_{i}-\sum_{i=1}^{n} \beta_{j} .
\end{aligned}
$$

This implies that

$$
\begin{aligned}
& \operatorname{Re}\left\{1+\frac{z F_{v_{1}, \ldots, v_{n}, \alpha_{1}, \ldots, \alpha_{n}, \beta_{1}, \ldots, \beta_{m}}^{\prime \prime}(z)}{F_{v_{1}, \ldots, v_{n}, \alpha_{1}, \ldots, \alpha_{n}, \beta_{1}, \ldots, \beta_{m}}^{\prime}(z)}\right\} \\
& =\sum_{i=1}^{n} \alpha_{i} \operatorname{Re}\left(\frac{z u_{v_{i}, b, c}^{\prime}(z)}{u_{v_{i}, b, c}(z)}\right)+\sum_{j=1}^{m} \beta_{j} \operatorname{Re}\left(\frac{D^{p+1} f_{j}(z)}{D^{p} f_{j}(z)}\right) \\
& +\left(1-\sum_{i=1}^{n} \alpha_{i}-\sum_{i=1}^{n} \beta_{j}\right) .
\end{aligned}
$$

Now, by using Lemma 2.1 for each $v_{i}$, where $i=1,2, \cdots n$, we obtain

$$
\operatorname{Re}\left\{1+\frac{z F_{v_{1}, \ldots, v_{n}, \alpha_{1}, \ldots, \alpha_{n}, \beta_{1}, \ldots, \beta_{m}}^{\prime \prime}(z)}{F_{v_{1}, \ldots, v_{n}, \alpha_{1}, \ldots, \alpha_{n}, \beta_{1}, \ldots, \beta_{m}}^{\prime}(z)}\right\}
$$




$$
\begin{aligned}
& \leq \sum_{i=1}^{n} \alpha_{i}\left(1+\frac{|c|\left(6 k_{i}-|c|\right)}{3\left(4 k_{i}-|c|\right)\left(3 k_{i}-|c|\right)}\right) \\
& +\sum_{j=1}^{m} \beta_{j} \gamma_{j}+\left(1-\sum_{i=1}^{n} \alpha_{i}-\sum_{i=1}^{n} \beta_{j}\right) \\
& =1+\sum_{i=1}^{n} \alpha_{i}\left(\frac{|c|\left(6 k_{i}-|c|\right)}{3\left(4 k_{i}-|c|\right)\left(3 k_{i}-|c|\right)}\right)+\sum_{j=1}^{m} \beta_{j}\left(\gamma_{j}-1\right) .
\end{aligned}
$$

Now consider the funnction

$$
\tau:\left(\frac{7|c|}{24}, \infty\right) \rightarrow \mathbb{R}
$$

defined by

$$
\tau(k)=\frac{|c|(6 k-|c|)}{3(4 k-|c|)(3 k-|c|)},
$$

is decreasing function

$$
\frac{|c|\left(6 k_{i}-|c|\right)}{3\left(4 k_{i}-|c|\right)\left(3 k_{i}-|c|\right)} \leq \frac{|c|(6 k-|c|)}{3(4 k-|c|)(3 k-|c|)} .
$$

Therefore

$$
\begin{aligned}
& \operatorname{Re}\left\{1+\frac{z F_{v_{1}, \ldots, v_{n}, \alpha_{1}, \ldots, \alpha_{n}, \beta_{1}, \ldots, \beta_{m}}^{\prime \prime}(z)}{F_{v_{1}, \ldots, v_{n}, \alpha_{1}, \ldots, \alpha_{n}, \beta_{1}, \ldots, \beta_{m}}^{\prime}(z)}\right\} \\
& \leq 1+\frac{|c|(6 k-|c|)}{3(4 k-|c|)(3 k-|c|)} \sum_{i=1}^{n} \alpha_{i}+\sum_{j=1}^{m} \beta_{j}\left(\gamma_{j}-1\right) .
\end{aligned}
$$

Since $1<1+\frac{|c|(6 k-|c|)}{3(4 k-|c|)(3 k-|c|)} \sum_{i=1}^{n} \alpha_{i}+\sum_{j=1}^{m} \beta_{j}\left(\gamma_{j}-1\right)<\frac{2^{p}+1}{2^{p-1}+1}$

therefore $F_{v_{1}, \ldots, v_{n}, \alpha_{1}, \ldots, \alpha_{n}, \beta_{1}, \ldots, \beta_{m}} \in N(\mu)$, where

$$
\mu=1+\frac{|c|(6 k-|c|)}{3(4 k-|c|)(3 k-|c|)} \sum_{i=1}^{n} \alpha_{i}+\sum_{j=1}^{m} \beta_{j}\left(\gamma_{j}-1\right) .
$$

Which completes the proof.

By setting $\beta_{1}=\beta_{2}=\ldots=\beta_{n}=0$ and $p=1$ in Theorem 3.1 we will obtain the result given below

Corollary 3.2. Let $v_{1}, \ldots, v_{n}, b \in \mathbb{R}, c \in \mathbb{C}$ and $k_{i}>\frac{7|c|}{24}$ with $k_{i}=v_{i}+$ $(b+2) / 2, i=1, \ldots, n$. Let $u_{v_{i}, b, c}: \mathcal{U} \rightarrow \mathbb{C}$ be defined as

$$
u_{v_{i}, b, c}(z)=2^{v} \sqrt{\pi} \Gamma\left(v+\frac{b+2}{2}\right) z^{\frac{(-1-v)}{2}} w_{v_{i}, b, c}(\sqrt{z}) .
$$

Suppose $v=\min \left\{v_{1}, v_{2}, \ldots, v_{n}\right\}$. Let $\alpha_{1}, \ldots, \alpha_{n}(i=1,2, \ldots, n)$ are positive real numbers and $f_{j}(z),(j=1,2, \ldots, m)$ be of the form of (1) is in class $N\left(p, \gamma_{j}\right)$. More over these numbers satisfy the relation 


$$
1<1+\frac{|c|(6 k-|c|)}{3(4 k-|c|)(3 k-|c|)} \sum_{i=1}^{n} \alpha_{i}<\frac{3}{2},
$$

then the function $F_{v_{1}, \ldots, v_{n}, \alpha_{1}, \ldots, \alpha_{n}, \beta_{1}, \ldots, \beta_{m}}: \mathcal{U} \rightarrow \mathbb{C}$ defined by 12$]$ is in $N(\mu)$, where

$$
\mu=1+\frac{|c|(6 k-|c|)}{3(4 k-|c|)(3 k-|c|)} \sum_{i=1}^{n} \alpha_{i} .
$$

The next theorem gives other sufficient conditions for the integral operator defined in (12). The key tool in the proof is Lemma 2.2 .

\section{Sufficient Conditions of Integral Operator Defined by Normalized Dini Function}

Theorem 4.1. Let $v_{1}, \ldots, v_{n}>\frac{-9+\sqrt{33}}{8}$, where $n \in \mathbb{N}$. Let $q_{v_{i}}: \mathcal{U} \rightarrow \mathbb{C}$ be defined as

$$
q_{v_{i}}(z)=2^{v_{i}-1} \Gamma\left(v_{i}+1\right) z^{1-\frac{v_{i}}{2}}\left(\left(2-v_{i}\right) J_{v_{i}}(\sqrt{z})+\sqrt{z} J_{v_{i}}^{\prime}(\sqrt{z})\right) .
$$

Suppose $k=\min \left\{k_{1}, k_{2}, \ldots, k_{n}\right\}, \alpha_{1}, \ldots, \alpha_{n}, \beta_{1}, \ldots, \beta_{m}$ are positive real numbers and let $f_{j}(z),(j=1,2, \ldots, m)$ be of the form of (1) is in class $N\left(p, \gamma_{j}\right)$. More over these numbers satisfy the relation

$$
1<1+\frac{4 v+9}{2\left(4 v^{2}+9 v+3\right)} \sum_{i=1}^{n} \alpha_{i}+\sum_{j=1}^{m} \beta_{j}\left(\gamma_{j}-1\right)<\frac{2^{p}+1}{2^{p-1}+1},
$$

then the function $F_{v_{1}, \ldots, v_{n}, \alpha_{1}, \ldots, \alpha_{n}, \beta_{1}, \ldots, \beta_{m}}: \mathcal{U} \rightarrow \mathbb{C}$ defined by 12 is in $N(\mu)$, where

$$
\mu=1+\frac{4 v+9}{2\left(4 v^{2}+9 v+3\right)} \sum_{i=1}^{n} \alpha_{i}+\sum_{j=1}^{m} \beta_{j}\left(\gamma_{j}-1\right) .
$$

Proof. We easily observe that $q_{v_{i}} \forall i=1,2, \cdots n$ are analytic and normalized of the form of $q_{v_{i}}(0)=q_{v_{i}}^{\prime}(0)-1=0$. Clearly $F_{v_{1}, \ldots, v_{n}, \alpha_{1}, \ldots, \alpha_{n}, \beta_{1}, \ldots, \beta_{m}}$ also analytic and normalized form of the form of

$$
F_{v_{1}, \ldots, v_{n}, \alpha_{1}, \ldots, \alpha_{n}, \beta_{1}, \ldots, \beta_{m}}(0)=F_{v_{1}, \ldots, v_{n}, \alpha_{1}, \ldots, \alpha_{n}, \beta_{1}, \ldots, \beta_{m}}^{\prime}(0)-1=0 .
$$

On the other hand, it is easy to see that

$$
F_{v_{1}, \ldots, v_{n}, \alpha_{1}, \ldots, \alpha_{n}, \beta_{1}, \ldots, \beta_{m}}(z)=\prod_{i=1}^{n}\left(\frac{q_{v_{i}}}{z}\right)^{\alpha_{i}} \prod_{i=1}^{n}\left(\frac{D^{p} f_{j}(z)}{z}\right)^{\beta_{j}} .
$$

Differentiating logarithmically, we get

$$
\begin{aligned}
& \frac{z F_{v_{1}, \ldots, v_{n}, \alpha_{1}, \ldots, \alpha_{n}, \beta_{1}, \ldots, \beta_{m}}^{\prime \prime}(z)}{F_{v_{1}, \ldots, v_{n}, \alpha_{1}, \ldots, \alpha_{n}, \beta_{1}, \ldots, \beta_{m}}^{\prime}(z)} \\
& =\sum_{i=1}^{n} \alpha_{i}\left(\frac{z q_{v_{i}}^{\prime}(z)}{q_{v_{i}}(z)}-1\right)+\sum_{j=1}^{m} \beta_{j}\left(\frac{D^{p+1} f_{j}(z)}{D^{p} f_{j}(z)}-1\right),
\end{aligned}
$$


or equivalently,

$$
\begin{aligned}
& 1+\frac{z F_{v_{1}, \ldots, v_{n}, \alpha_{1}, \ldots, \alpha_{n}, \beta_{1}, \ldots, \beta_{m}}^{\prime \prime}(z)}{F_{v_{1}, \ldots, v_{n}, \alpha_{1}, \ldots, \alpha_{n}, \beta_{1}, \ldots, \beta_{m}}^{\prime}(z)} \\
& =\sum_{i=1}^{n} \alpha_{i}\left(\frac{q_{v_{i}}^{\prime}(z)}{q_{v_{i}}(z)}\right)+\sum_{j=1}^{m} \beta_{j}\left(\frac{D^{p+1} f_{j}(z)}{D^{p} f_{j}(z)}\right)+1-\sum_{i=1}^{n} \alpha_{i}-\sum_{i=1}^{n} \beta_{j} .
\end{aligned}
$$

This implies that

$$
\begin{aligned}
& \operatorname{Re}\left\{1+\frac{z F_{v_{1}, \ldots, v_{n}, \alpha_{1}, \ldots, \alpha_{n}, \beta_{1}, \ldots, \beta_{m}}^{\prime \prime}(z)}{F_{v_{1}, \ldots, v_{n}, \alpha_{1}, \ldots, \alpha_{n}, \beta_{1}, \ldots, \beta_{m}}^{\prime}(z)}\right\} \\
& =\sum_{i=1}^{n} \alpha_{i} \operatorname{Re}\left(\frac{z q_{v_{i}}^{\prime}(z)}{q_{v_{i}}(z)}\right)+\sum_{j=1}^{m} \beta_{j} \operatorname{Re}\left(\frac{D^{p+1} f_{j}(z)}{D^{p} f_{j}(z)}\right)+\left(1-\sum_{i=1}^{n} \alpha_{i}-\sum_{i=1}^{n} \beta_{j}\right) .
\end{aligned}
$$

Now, by using Lemma 2.2 for each $v_{i}$, where $i=1,2, \cdots n$, we obtain

$$
\begin{aligned}
& \operatorname{Re}\left\{1+\frac{z F_{v_{1}, \ldots, v_{n}, \alpha_{1}, \ldots, \alpha_{n}, \beta_{1}, \ldots, \beta_{m}}^{\prime \prime}(z)}{F_{v_{1}, \ldots, v_{n}, \alpha_{1}, \ldots, \alpha_{n}, \beta_{1}, \ldots, \beta_{m}}^{\prime}(z)}\right\} \\
& \leq \sum_{i=1}^{n} \alpha_{i}\left(1+\frac{4 v+9}{2\left(4 v^{2}+9 v+3\right)}\right) \\
& +\sum_{j=1}^{m} \beta_{j} \gamma_{j}+\left(1-\sum_{i=1}^{n} \alpha_{i}-\sum_{i=1}^{n} \beta_{j}\right) \\
& =1+\sum_{i=1}^{n} \alpha_{i}\left(\frac{4 v+9}{2\left(4 v^{2}+9 v+3\right)}\right)+\sum_{j=1}^{m} \beta_{j}\left(\gamma_{j}-1\right) .
\end{aligned}
$$

Now as it is clear that the function

$$
\phi(v):\left(\frac{-9+\sqrt{33}}{8}, \infty\right) \rightarrow \mathbb{R},
$$

defined by

$$
\phi(v)=\frac{4 v+9}{2\left(4 v^{2}+9 v+3\right)},
$$

is decreasing function. Therefore

$$
\frac{4 v_{i}+9}{2\left(4 v_{i}^{2}+9 v_{i}+3\right)} \leq \frac{4 v+9}{2\left(4 v^{2}+9 v+3\right)} .
$$

Therefore

$$
\begin{aligned}
& \operatorname{Re}\left\{1+\frac{z F_{v_{1}, \ldots, v_{n}, \alpha_{1}, \ldots, \alpha_{n}, \beta_{1}, \ldots, \beta_{m}}^{\prime \prime}(z)}{F_{v_{1}, \ldots, v_{n}, \alpha_{1}, \ldots, \alpha_{n}, \beta_{1}, \ldots, \beta_{m}}^{\prime}(z)}\right\} \\
& \leq 1+\frac{4 v+9}{2\left(4 v^{2}+9 v+3\right)} \sum_{i=1}^{n} \alpha_{i}+\sum_{j=1}^{m} \beta_{j}\left(\gamma_{j}-1\right) .
\end{aligned}
$$


Since $1<1+\frac{4 v+9}{2\left(4 v^{2}+9 v+3\right)} \sum_{i=1}^{n} \alpha_{i}+\sum_{j=1}^{m} \beta_{j}\left(\gamma_{j}-1\right)<\frac{2^{p}+1}{2^{p-1}+1}$, therefore

$$
F_{v_{1}, \ldots, v_{n}, \alpha_{1}, \ldots, \alpha_{n}, \beta_{1}, \ldots, \beta_{m}} \in N(\mu)
$$

where

$$
\mu=1+\frac{4 v+9}{2\left(4 v^{2}+9 v+3\right)} \sum_{i=1}^{n} \alpha_{i}+\sum_{j=1}^{m} \beta_{j}\left(\gamma_{j}-1\right)
$$

which completes the proof.

By setting $\beta_{1}=\beta_{2}=\ldots=\beta_{n}=0$ and $p=1$ in Theorem 4.1 we will obtain the result given below

Corollary 4.2. Let $v_{1}, \ldots, v_{n}>\frac{-9+\sqrt{33}}{8}$, where $n \in \mathbb{N}$. Let $q_{v_{i}}: \mathcal{U} \rightarrow \mathbb{C}$ be defined as

$$
q_{v_{i}}(z)=2^{v_{i}-1} \Gamma\left(v_{i}+1\right) z^{1-\frac{v_{i}}{2}}\left(\left(2-v_{i}\right) J_{v_{i}}(\sqrt{z})+\sqrt{z} J_{v_{i}}^{\prime}(\sqrt{z})\right) .
$$

Suppose $v=\min \left\{v_{1}, v_{2}, \ldots, v_{n}\right\}$. Let $\alpha_{1}, \ldots, \alpha_{n}(i=1,2, \ldots, n)$ are positive real numbers and $f_{j}(z),(j=1,2, \ldots, m)$ be of the form of (1) is in class $N\left(p, \gamma_{j}\right)$. More over these numbers satisfy the relation

$$
1<1+\frac{4 v+9}{2\left(4 v^{2}+9 v+3\right)} \sum_{i=1}^{n} \alpha_{i}<\frac{3}{2},
$$

then the function $F_{v_{1}, \ldots, v_{n}, \alpha_{1}, \ldots, \alpha_{n}, \beta_{1}, \ldots, \beta_{m}}: \mathcal{U} \rightarrow \mathbb{C}$ defined by 12 is in $N(\mu)$, where

$$
\mu=1+\frac{4 v+9}{2\left(4 v^{2}+9 v+3\right)} \sum_{i=1}^{n} \alpha_{i}
$$

\section{Competing Interests}

The author(s) do not have any competing interests in the manuscript.

\section{REFERENCES}

1. Salagean, G. S. (1983). Subclasses of univalent functions. In Complex Analysis-Fifth Romanian-Finnish Seminar (pp. 362-372). Springer, Berlin, Heidelberg.

2. Uralegaddi, B. A., Ganigi, M. D., \& Sarangi, S. M. (1994). Univalent functions with positive coefficients. Tamkang J. Math, 25(3), 225-230.

3. Baricz, Á. R. P. Á. D. (2006). Bessel transforms and Hardy space of generalized Bessel functions. Mathematica, 48(71), 127-136.

4. Baricz, Á. (2010). Generalized Bessel functions of the first kind. Springer.

5. Bansal, D., \& Prajapat, J. K. (2016). Certain geometric properties of the Mittag-Leffler functions. Complex Variables and Elliptic Equations, 61(3), 338-350.

6. Orhan, H., \& Yagmur, N. (2014). Geometric properties of generalized Struve functions. Annals of the Alexandru Ioan Cuza University-Mathematics.

7. Raza, M., Din, M. U., \& Malik, S. N. (2016). Certain geometric properties of normalized Wright functions. Journal of Function Spaces, 2016,Article ID 1896154, 8 pages.

8. Srivastava, H. M., Din, M. U.,\& Raza, M., Univalence of Certain Integral Operators Involving Generalized Struve functions. (accepted). 
9. Baricz, Á., Deniz, E., \& Yaǵmur, N. (2016). Close-to-convexity of normalized Dini functions. Mathematische Nachrichten, 289(14-15), 1721-1726.

10. Baricz,Á ., Ponnusamy, S., \& Singh, S. (2016). Modified Dini functions: monotonicity patterns and functional inequalities. Acta Mathematica Hungarica, 149(1), 120-142.

11. Breaz, D. (2008). Certain Integral Operators on the Classes and. Journal of Inequalities and Applications, 2008(1), 719354.

12. Din, M. U, Raza, M, \& Deniz, E. Sufficient Conditions for Univalence of Integral Operators Involving Dini Functions. (Submitted).

13. Deniz, E., Orhan, H., \& Srivastava, H. M. (2011). Some sufficient conditions for univalence of certain families of integral operators involving generalized Bessel functions. Taiwanese J. Math, 15(2), 883-917.

14. Struve, H. (1882). Beitrag zur Theorie der Diffraction an Fernrhren. Annalen der Physik, 253(13), 1008-1016.

15. Pascu, N. N., \& Pescar, V. (1990). On the integral operators of Kim-Merkes and Pfaltzgraff. Mathematica (Cluj), 32(55), 185-192.

16. Porwal, S. (2011). Mapping properties of an integral operator. Acta Universitatis Apulensis, (27), 151-155.

17. Porwal, S., \& Breaz, D. (2014). Mapping properties of an integral operator involving Bessel functions. In Analytic Number Theory, Approximation Theory, and Special Functions (pp. 821-826). Springer New York.

18. Porwal, S., \& Kumar, M. (2017). Mapping properties of an integral operator involving Bessel functions. Afrika Matematika, 28(1-2), 165-170.

Muhey U Din

Department of Mathematics, Government College University Faisalabad, Pakistan.

e-mails: muheyudin@yahoo.com

Mohsan Raza

Department of Mathematics, Government College University Faisalabad, Pakistan.

e-mail: mohsan976@yahoo.com

Saddaf Noreen

Department of Mathematics, Government College University Faisalabad, Pakistan. e-mail: saddafnoreen@yahoo.com 